\title{
La méthode EMERGY : principes et application en analyse environnementale des systèmes agricoles et de production animale
}

\author{
A. WILFART $T^{1,2}$, M.-S. CORSON ${ }^{1,2}$, J. AUBIN ${ }^{1,2}$ \\ ${ }^{1}$ INRA, UMR1069 Sol Agro et hydrosystème Spatialisation, F-35000 Rennes, France \\ 2 Agrocampus Ouest, UMR Sol Agro et hydrosystème Spatialisation, F-35000 Rennes, France \\ Courriel : aurelie.wilfart@rennes.inra.fr
}

La mise en œuvre de systèmes agricoles et de production animale plus économes en intrants et moins polluants nécessite des méthodes permettant d'évaluer leurs impacts environnementaux. La méthode Emergy, qui quantifie toutes les formes d'énergie nécessaires à la réalisation d'un service ou d'un produit, apparaît comme une méthode intéressante pour replacer un système agricole dans son environnement et analyser les interactions existant entre les deux.

L'agriculture procède d'une combinaison entre les intrants provenant du capital naturel (sol, énergie solaire, pluie, énergie fossile) et les intrants provenant du capital créé par l'homme (engrais, semences, pesticides) pour générer les produits voulus (van der Werf et Petit 2002). Le système d'élevage, tel que défini par Landais (1987), correspond à cette définition puisqu'il s'agit «d'un ensemble d'éléments en interaction dynamique organisé par l'homme en vue de valoriser des ressources par l'intermédiaire d'animaux domestiques pour en obtenir des productions variées ou pour répondre à d'autres objectifs». De nos jours, un des défis de l'agriculture et de l'élevage, est de trouver comment nourrir une population croissante tout en diminuant l'utilisation des sources d'énergie fossile et des ressources environnementales disponibles en quantités limitées. Dans cette perspective, les systèmes agricoles doivent évoluer et devenir plus efficients et plus respectueux de l'environnement. Pour cela, ils devront intégrer plus de ressources renouvelables (notamment énergétiques), moins de produits chimiques, et exploiter au mieux le fonctionnement des écosystèmes sans altérer leur renouvellement. Une telle agriculture peut être qualifiée d'écologiquement intensive (Griffon 2010). Afin d'accompagner l'évolution des systèmes agricoles, il est nécessaire de pouvoir évaluer leur performance et leur niveau de pression sur l'environnement.

Dans les systèmes de production agricole mobilisant beaucoup les ressources naturelles, il est difficile de mener des analyses environnementales classiques (comme les Analyses de Cycle de Vie (ACV), ou les analyses énergétiques de type Planète) car elles considèrent essentiellement les moyens injectés dans le système par l'homme. En effet, l'indicateur «énergie» de l'ACV environnementale ne prend en compte que les énergies non renouvelables utilisées directement ou indirectement dans le cycle de vie d'un produit. Dans ces méthodes, les énergies dites renouvelables utilisées dans la production d'électricité ne sont abordées que sous l'angle du matériel utilisé pour les exploiter. Par exemple, le vent à l'origine du fonctionnement des éoliennes n'est pas pris en compte. De plus, ces indicateurs ne considèrent pas l'énergie fournie par l'homme ou les animaux (pas de prise en compte directe du travail) pour réaliser un produit, ni l'énergie issue du soleil ou de la pluie permettant la croissance de la biomasse dans les systèmes agricoles. La méthode Emergy (Odum 1996) est un outil prometteur pour évaluer les systèmes agricoles qui sont des systèmes se situant à l'interface de la nature et des contributions humaines (Castellini et al 2006, Pizzigallo et al 2008). Elle permet d'évaluer chaque composante d'un système telle que l'utilisation des ressources achetées, d'origine naturelle, des services et les productions des systèmes (Martin et al 2006) sur la base d'une unité énergétique commune (l'énergie solaire). Au cours de cet article, le concept d'Emergy, sa symbolique et son utilisation seront présentés en s'appuyant sur l'exemple du fonctionnement d'un système de production de poissons en étang en France.

\section{1 / La méthode d'évaluation Emergy}

\section{1 / Le concept d'Emergy}

La méthode Emergy est basée sur les principes de la thermodynamique. L'Emergy est définie comme étant l'énergie solaire disponible utilisée directement et indirectement pour réaliser un service ou un produit (Odum 1996). Cette méthode a été développée pour évaluer l'ensemble des besoins en énergie pour obtenir un produit (Paoli et al 2008). Par exemple, la production de poissons en étang nécessite de l'eau, du soleil pour le développement de la biomasse, du sol, mais aussi des fertilisants, du matériel et des services tel que le travail humain. Cependant, les joules issus d'un type d'énergie (ex. l'eau) ne sont pas équivalents à ceux d'un autre type d'énergie (ex. les fertilisants) dans 
Figure 1. Le principe de la méthode Emergy, exemple de la production de poissons d'étang (d'après Ortega 2008).
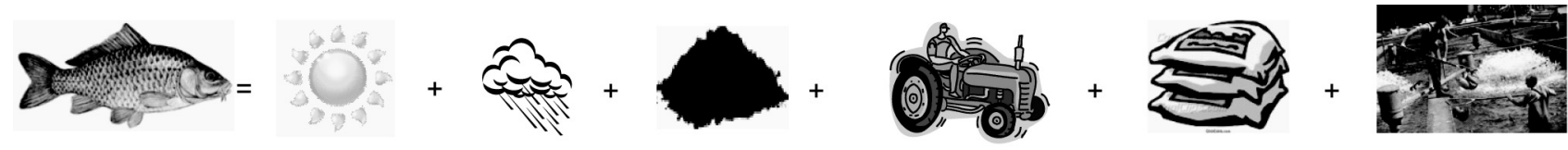

Soleil

Sol

Machines

Intrants

$\neq$

Services

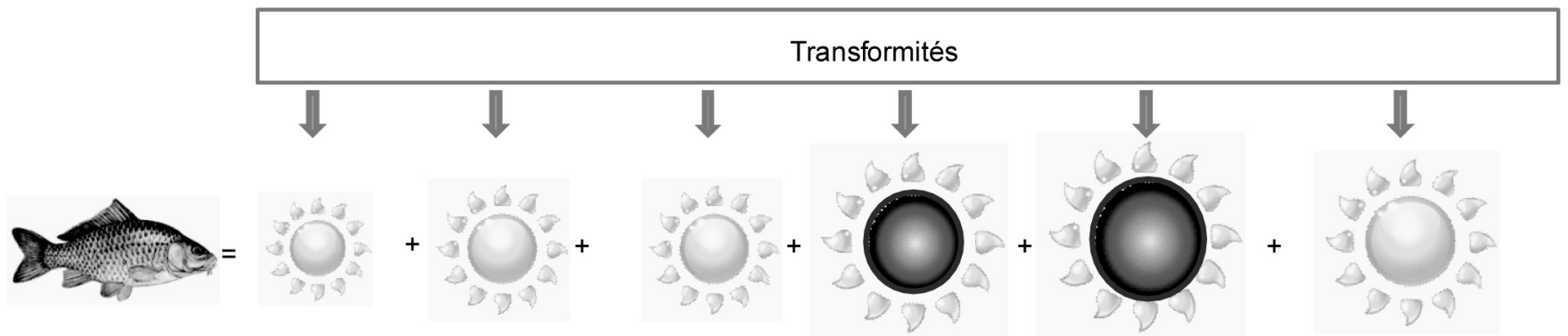

leur capacité à induire un même processus (figure 1).

L'Emergy est donc une méthode d'analyse quantitative qui exprime les ressources non-monétaires (ressources naturelles telles que le soleil, la pluie, le vent...), les ressources monétaires, les services et les produits dans une unité commune (Bastianoni et al 2001). Cette unité est exprimée en quantité d'énergie solaire utilisée (Odum 1996). Elle est appelée Emergy solaire et s'exprime en joules d'Emergy solaire dont l'abréviation est «pour sej» «pour solar emjoule». L'analyse Emergy caractérise donc tous les produits et les services en équivalent énergie solaire qui représente la quantité d'énergie qui serait nécessaire pour réaliser une tâche particulière si les radiations solaires étaient les seuls intrants. Le concept considère la terre comme un système ayant comme seuls intrants énergétiques majeurs et constants l'énergie solaire, la chaleur interne de la terre et l'énergie marémotrice. Ainsi, tous les systèmes vivants sont connectés au sein d'un réseau de circulation de l'énergie convertissant l'énergie issue de ces trois sources (qui existe en grande quantité mais qui est peu concentrée - i.e. de qualité inférieure) en une énergie de qualité supérieure (i.e. une énergie plus concentrée et plus facilement exploitable) et en énergie thermique dégradée. Toutes les autres formes d'énergie existant sur terre peuvent être dérivées de ces 3 sources principales par le biais de transformations énergétiques. Puisque l'énergie solaire est l'intrant énergétique majoritaire sur terre, toutes les autres formes d'énergie sont converties en équivalent solaire afin d'être exprimées dans une unité commune. L'économie peut être aussi comptabilisée dans ce réseau de flux d'énergie. Dans ce cas, elle est considérée comme étant «la richesse directe et indirecte» provenant des ressources environnementales mesurées par l'Emergy (Odum 1996). Le flux écono- mique sera exprimé en unité monétaire $(€, \$, \ldots)$, la transformité en sej/unité monétaire et le flux d'Emergy en sej.

\section{2 / Les transformités}

Les transformités (ou coefficients de transformation) estiment la quantité d'Emergy (en sej) requise directement ou indirectement pour produire une unité d'un bien ou d'un service. Elles correspondent au ratio de l'Emergy nécessaire pour produire un flux ou une unité de stock, sur l'énergie réelle de ce flux ou de ce stock (Ulgiati et Brown 2002). Ainsi, la transformité solaire d'un produit est le ratio de son Emergy solaire sur l'énergie qu'il contient (en joules) et se mesure donc en sej/J. La transformité d'un produit correspond donc à la variable $\tau$ de l'équation suivante : $\mathrm{Y}=\tau \times \mathrm{E}$ où $\mathrm{Y}$ est l'Emergy et $\mathrm{E}$ le contenu énergétique du produit. Elle indique la position hiérarchique d'un

produit sur une échelle thermodynamique. Plus elle sera élevée, plus il y aura eu de transformation et de création d'Emergy associées à ce produit. Par exemple, le flux d'Emergy total nécessaire pour produire $1,7 \times 10^{11}$ joules de poissons en étang (soit 35 tonnes) est de $1,14 \times 10^{18}$ sej. La transformité associée à cette production de poissons est donc de $6,9 \times 10^{6}$ sej par joule. Ainsi, les transformités permettent-elles de convertir chaque flux d'un système en Emergy solaire et de les exprimer dans une unité commune. Ceci permet une comparaison entre flux et entre systèmes. Lorsque des systèmes ayant des productions équivalentes sont comparés, une plus forte transformité indique que moins de produits sont obtenus avec la même quantité d'Emergy investie ou que plus de ressources sont nécessaires pour obtenir le même niveau de production. Des exemples de valeurs de transformités sont donnés dans le tableau 1 .

Tableau 1. Exemples de valeur de transformités (Odum 1996 modifiée par Odum 2000, Castellini et al 2006, Cavalett et al 2006).

\begin{tabular}{|l|c|}
\hline Item & Transformité (sej/J) \\
\hline Ensoleillement direct & 1 \\
\hline Vent & 2513 \\
\hline Pluie & 30574 \\
\hline Charbon & 67200 \\
\hline Pétrole brut & 90720 \\
\hline Matière organique de la couche superficielle du sol & 124320 \\
\hline Électricité & 291784 \\
\hline Engrais azoté & 3124800 \\
\hline Saumon (Salmon salar) & 9700000 \\
\hline Engrais phosphaté & 16968000 \\
\hline Maïs non irrigué & 888000000 \\
\hline Tourteau de soja & 3260000000 \\
\hline Information génétique, espèce d'arbre & 1219680000000 \\
\hline Information génétique, ADN humain & 24696000000000000 \\
\hline
\end{tabular}


Figure 2. Exemples de symboles utilisés dans les diagrammes de la méthode Emergy (d'après Odum 1996).

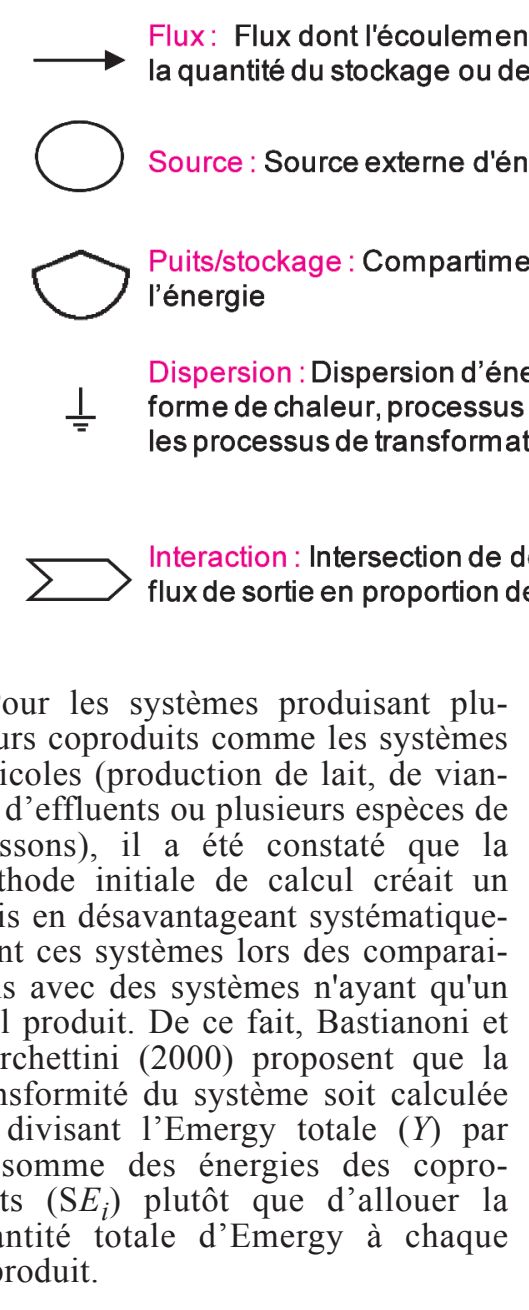

L'énergie solaire étant la base de tous les calculs en Emergy, la transformité de l'énergie solaire absorbée par la terre est de 1. La plupart des transformités est calculée à partir du flux annuel d'Emergy sur la terre (Odum 2000). Si les transformités ne sont pas disponibles dans la littérature ou non adaptées au contexte du travail, Odum (1996) propose plusieurs façons de les calculer (Encadré 1). Il est donc nécessaire lors des analyses Emergy d'indiquer l'origine de chaque transformité utilisée.

Il existe actuellement des folios regroupant les transformités de base édités par le centre de politique environnementale de Floride (Center for Environmental Policy, Gainsville, Florida, USA : http://www.cep.ees. ufl.edu/publications.asp). Une réévaluation des quantités d'énergie solaire nécessaires aux phénomènes biologiques et économiques a été réalisée par Odum en 2000. Elle implique qu'un facteur de correction de 1,68 doit être appliqué aux transformités publiées avant 2000.

\section{2 / Mise en ouvre de la méthode, application à l'a- nalyse de la production de poissons en étang}

\section{1 / Les différentes étapes de la mise en ouvre}

La première étape d'une analyse Emergy consiste à réaliser un diagramme énergétique du système. Cette étape est nécessaire afin d'organiser les différentes relations existantes entre les principaux processus du système à étudier. Ce diagramme est construit à partir d'un langage de modélisation utilisant des symboles spécifiques développés par Odum (1983, 1996). Des exemples de symboles sont représentés dans la figure 2 .

La deuxième étape consiste à organiser les différents flux représentés dans les diagrammes dans des tableaux d'évaluation. Ces tableaux permettront de calculer les indicateurs Emergy. La dernière étape consiste en l'interprétation des résultats.

\section{2 / Le diagramme énergétique et la classification des flux}

Le diagramme permet d'organiser les relations existantes entre les principaux composants et les processus du système. Cela permet aussi de décrire l'écosystè-

Encadré 1. Méthodes de calcul des transformités (Odum 1996).

- Les transformités des principaux flux d'énergie issus de l'environnement (ex. vent, pluie, terre) peuvent être calculées à partir du réseau énergétique de la géo-biosphère :

- Soit ces flux sont considérés comme des coproduits des trois sources d'énergie principales (énergie solaire, chaleur interne de la terre et énergie marémotrice) et dans ce cas là le flux total d'Emergy alimentant le réseau est divisé par l'énergie de chaque flux : le flux d'Emergy est le même pour chaque flux principal mais chaque flux a sa propre transformité.

- Soit ces flux sont considérés comme des fractions du flux total d'Emergy ce qui entraîne que le flux d'Emergy par branche sera différent, mais que la transformité sera identique.

- Les transformités, concernant les produits dits environnementaux (ex. eau souterraine, sol...) ou les produits manufacturés et services, peuvent être calculées à partir de l'analyse des sous-systèmes de production et de transformation de l'énergie.

- Dans les cas, où l'Emergy est stockée dans des réserves, comme le sol ou l'eau souterraine, les transformités peuvent être calculées en prenant en compte le temps d'accumulation et/ou le turn-over si celui si est supérieur à un an.

II est possible aussi après analyse des sous-systèmes d'obtenir la transformité d'un produit en combinant d'autres transformités par exemple pour déterminer la transformité de l'électricité qui est le résultat d'un mélange de différentes sources (ex. énergie nucléaire, centrale hydroélectrique, panneaux photovoltaïques, biomasse...).

- Lorsque les analyses Emergy ne sont pas disponibles, il est possible de déterminer les transformités en évaluant les transformations de l'énergie issues d'autres méthodes d'analyses environnementales (ex. conversion des données de charges en flux d'énergie disponible lors des écoulements de rivières). 
Figure 3. Diagramme Emergy d'un système de production de poissons en étang.

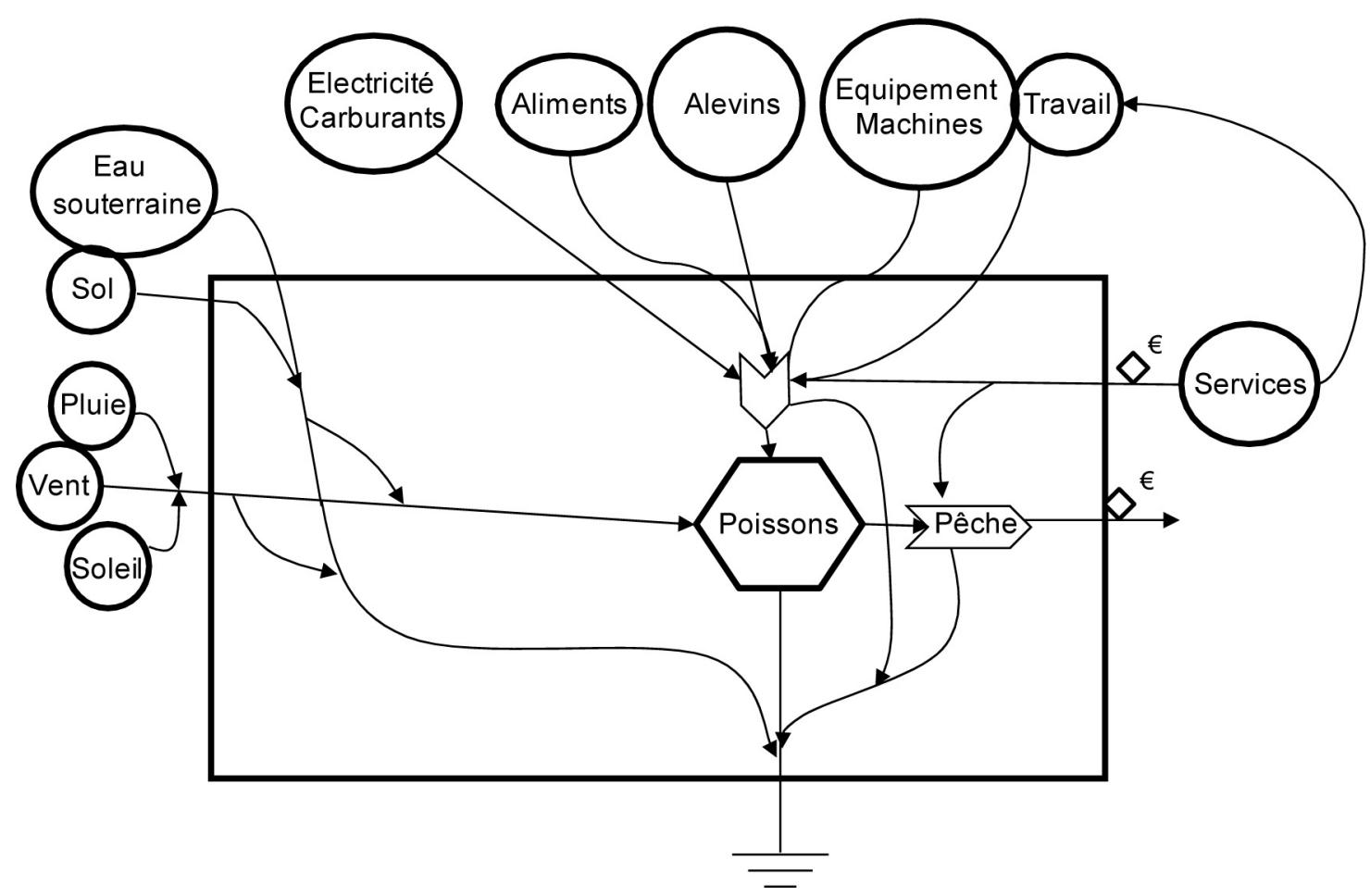

me sur des bases environnementales tout en le reliant au monde économique (Cavalett et al 2006). La figure 3 représente un système de production de poissons en étang, traduit dans le symbolisme proposé par Odum (1983, 1996). Les flux sont classiquement représentés de la manière suivante : à gauche, les flux issus de la nature (I), en haut les intrants provenant de l'activité humaine (F), à droite les interactions avec le monde économique. Ce diagramme permet donc de classer les différents flux selon leur origine. Les flux issus de l'activité humaine (i.e. flux achetés, $(\mathrm{F})$ ) seront classés en matériaux $(\mathrm{M})$ et servi-

Tableau 2. Classification des flux Emergy utilisée dans les études environnementales.

\begin{tabular}{|c|c|}
\hline Intrants et Services & Description \\
\hline I: Contribution de la nature & Ressources locales, gratuites $\mathrm{R}+\mathrm{N}$ \\
\hline $\begin{array}{l}\mathrm{R} \text { : Ressources renouvelables } \\
\text { provenant de la nature }\end{array}$ & $\begin{array}{l}\text { Soleil, pluie, vent, marée } \\
\left(\% \mathrm{R}_{\mathrm{R}}=100 \%\right)\end{array}$ \\
\hline $\begin{array}{l}\mathrm{N} \text { : Ressources non renouvelables } \\
\text { provenant de la nature }\end{array}$ & $\begin{array}{l}\text { Ressources à renouvellement lent (ex. } \\
\text { sol, biodiversité, eaux des nappes), } \\
\% \mathrm{R}_{\mathrm{N}}=0 \%\end{array}$ \\
\hline F : Ressource économique & Ressources payantes, $\mathrm{F}=\mathrm{M}+\mathrm{S}$ \\
\hline $\mathrm{M}:$ Matériaux & $M=M_{R}+M_{N}$ \\
\hline $\begin{array}{l}M_{R}: \text { Matériaux et énergie } \\
\text { renouvelables }\end{array}$ & Part renouvelable du matériel $\left(\% \mathrm{R}_{\mathrm{M}}\right)$ \\
\hline $\begin{array}{l}\mathrm{M}_{N} \text { : Matériaux et énergie non } \\
\text { renouvelables }\end{array}$ & $\begin{array}{l}\text { Part non-renouvelable du matériel } \\
\left(100-\% R_{M}\right)\end{array}$ \\
\hline S: Services & $\mathrm{S}=\mathrm{S}_{\mathrm{R}}+\mathrm{S}_{\mathrm{N}}$ \\
\hline$S_{R}:$ Services renouvelables & Part renouvelable des services $\left(\% \mathrm{R}_{\mathrm{S}}\right)$ \\
\hline$S_{N}$ : Services non renouvelables & $\begin{array}{l}\text { Part non renouvelable des services } \\
\left(100-\% R_{S}\right)\end{array}$ \\
\hline $\mathrm{Y}:$ Emergy totale & $Y=I+F$ \\
\hline
\end{tabular}

mité, le flux d'Emergy. Le pourcentage de chaque classe (R, N...) est de plus en plus fréquemment mentionné afin d'affiner le calcul des indicateurs.

Le tableau 3 représente le tableau d'évaluation issu du diagramme énergétique d'un système de production de poissons en étang en France. Les flux identifiés dans le diagramme énergétique sont répartis en fonction de leur origine (issus de la nature, intrants, services). La colonne 3 permet d'indiquer l'unité dans laquelle est exprimé le flux $(\mathrm{J}, \mathrm{kg}, €)$ et la colonne 4 indique la quantité annuelle de ce flux. La colonne 5 indique la transformité associée au flux et grâce à laquelle le flux sera exprimé en joule d'Emergy solaire par an (sej/an, colonne 6). La colonne 7 indique la référence de la transformité et la colonne 8 permet d'indiquer quelle est la part renouvelable dans le flux d'Emergy. Ainsi, les intrants renouvelables issus de la nature ont un pourcentage de cent alors que les intrants non renouvelables issus de la nature ont un pourcentage nul. Pour les autres intrants, la part renouvelable dépendra de la proportion d'Emergy renouvelable nécessaire à leur obtention. Le bas du tableau indique la quantité totale d'Emergy $(Y)$ entrée dans le système ainsi que la masse et l'énergie de tous les produits.

\section{4 / Les indicateurs disponibles}

Le tableau 4 présente une sélection des indicateurs Emergy proposés par Odum (1996) et revus par Cavalett et al 
Tableau 3. Tableau d'évaluation d'un système de production de poissons en étang.

\begin{tabular}{|c|c|c|c|c|c|c|c|}
\hline Note & Item & Unité & $\begin{array}{l}\text { Flux annuel } \\
\text { (unitélan) }\end{array}$ & $\begin{array}{l}\text { Transformité, T } \\
\text { (sej/unité) }\end{array}$ & $\begin{array}{l}\text { Emergy solaire } \\
\qquad \begin{array}{c}\text { (sej/an) } \\
=\mathrm{x} \times \text { Flux }\end{array}\end{array}$ & $\begin{array}{l}\text { Référence } \\
\text { Transformité }\end{array}$ & $\begin{array}{c}\text { Part de } \\
\text { ressources } \\
\text { renouvelables } \\
(\% \mathrm{R})\end{array}$ \\
\hline \multicolumn{8}{|c|}{ Intrants renouvelables issus de la nature (R) } \\
\hline 1 & Ensoleillement & J & $3,9 \times 10^{15}$ & 1 & $3,9 \times 10^{15}$ & Odum (1996) & 100 \\
\hline 2 & Vent & J & $3,1 \times 10^{11}$ & $2,5 \times 10^{3}$ & $7,75 \times 10^{14}$ & Odum (2000) & 100 \\
\hline 3 & Pluie & $\mathrm{J}$ & $5,0 \times 10^{12}$ & $2,6 \times 10^{4}$ & $1,30 \times 10^{17}$ & Odum (2000) & 100 \\
\hline \multicolumn{8}{|c|}{ Intrants non renouvelables issus de la nature $(\mathrm{N})$} \\
\hline 4 & Sol & $\mathrm{J}$ & $2,7 \times 10^{8}$ & $1,2 \times 10^{5}$ & $3,33 \times 10^{13}$ & $\begin{array}{c}\text { Brandt-Williams } \\
(2002)\end{array}$ & 0 \\
\hline 5 & $\begin{array}{c}\text { Eau } \\
\text { souterraine }\end{array}$ & $J$ & $2,2 \times 10^{11}$ & $2,6 \times 10^{5}$ & $5,72 \times 10^{16}$ & $\begin{array}{l}\text { Bastianoni et } \\
\text { Marchettinni } \\
(2000)\end{array}$ & 0 \\
\hline \multicolumn{8}{|c|}{ Intrants achetés (M) } \\
\hline 6 & Diesel & $\mathrm{J}$ & $1,1 \times 10^{11}$ & $1,9 \times 10^{5}$ & $2,09 \times 10^{16}$ & Odum (2000) & 5 \\
\hline 7 & Electricité & $J$ & $7,7 \times 10^{11}$ & $2,7 \times 10^{5}$ & $2,08 \times 10^{17}$ & $\begin{array}{c}\text { Brandt-Williams } \\
(2002)\end{array}$ & 12 \\
\hline 8 & Béton & $\mathrm{kg}$ & $1,8 \times 10^{4}$ & $4,4 \times 10^{11}$ & $7,92 \times 10^{15}$ & Haukoos (1994) & 10 \\
\hline 9 & Blé & $\mathrm{J}$ & $4,7 \times 10^{11}$ & $1,2 \times 10^{6}$ & $5,64 \times 10^{17}$ & calculé & 20 \\
\hline 10 & Alevins & $\mathrm{kg}$ & $5 \times 10^{2}$ & $6,9 \times 10^{12}$ & $3,45 \times 10^{15}$ & $\begin{array}{c}\text { Cavalett et al } \\
2007\end{array}$ & 5 \\
\hline 11 & Chaux & $g$ & $9,6 \times 10^{6}$ & $1,7 \times 10^{9}$ & $1,63 \times 10^{16}$ & $\begin{array}{c}\text { Brown et Ulgiati } \\
(2004)\end{array}$ & 5 \\
\hline 12 & Bois & $J$ & $9,2 \times 10^{11}$ & $1,1 \times 10^{4}$ & $1,01 \times 10^{16}$ & $\begin{array}{c}\text { Odum et Brown } \\
(2000)\end{array}$ & 5 \\
\hline 13 & Machine & $\mathrm{kg}$ & $4,1 \times 10^{1}$ & $1,1 \times 10^{7}$ & $4,51 \times 10^{8}$ & Bergquist (2008) & 10 \\
\hline \multicolumn{8}{|c|}{ Travail et service (S) } \\
\hline 14 & Travail & $J$ & $8,2 \times 10^{9}$ & $1,2 \times 10^{6}$ & $1,00 \times 10^{17}$ & $\begin{array}{c}\text { Brandt-Williams } \\
(2002)\end{array}$ & 50 \\
\hline \multicolumn{2}{|c|}{ Emergy totale $(Y)$} & & & & $1,1 \times 10^{18}$ & & \\
\hline \multicolumn{8}{|c|}{ Sorties } \\
\hline & Carpes & $\mathrm{kg}$ & 5250 & & & & \\
\hline & & $\mathrm{J}$ & $2,8 \times 10^{10}$ & & & & \\
\hline & Sandres & $\mathrm{kg}$ & 1750 & & & & \\
\hline & & $\mathrm{J}$ & $9,5 \times 10^{9}$ & & & & \\
\hline & Tanches & $\mathrm{kg}$ & 3500 & & & & \\
\hline & & $\mathrm{J}$ & $1,3 \times 10^{10}$ & & & & \\
\hline & Gardons & $\mathrm{Kg}$ & 17500 & & & & \\
\hline & & $J$ & $8,9 \times 10^{10}$ & & & & \\
\hline & Brochets & $\mathrm{kg}$ & 5250 & & & & \\
\hline & & $\mathrm{J}$ & $1,9 \times 10^{10}$ & & & & \\
\hline & Perches & $\mathrm{kg}$ & 1750 & & & & \\
\hline & & $\mathrm{J}$ & $6,7 \times 10^{9}$ & & & & \\
\hline & Transformité & Sej/J & $6,90 \times 10^{6}$ & & & & \\
\hline & & Sej/kg & $3,26 \times 10^{13}$ & & & & \\
\hline
\end{tabular}

(2006), utilisés pour évaluer les performances d'un système. Ils permettent d'évaluer son efficacité énergétique, ses impacts sur l'environnement et donc sa durabilité environnementale.

Le pourcentage d'Emergy renouvelable «percentage of renewability» $(\% \mathrm{R})$ mesure le degré d'Emergy renouvelable par rapport à l'Emergy totale transitant dans le système. Sur le long terme, les systèmes ayant un \% élevé sont considérés comme étant plus durables sur le plan environnemental et pouvant réagir plus rapidement à un stress économique que les systèmes se reposant sur une part élevée de ressources non renouve- lables (Brown et Ulgiati 2004). C'est ce pourcentage qui est pris en compte pour évaluer la partie renouvelable (R) de chaque flux.

La charge environnementale «Environmental Loading Ratio» (ELR) est le ratio entre l'Emergy issue des ressour- 
Tableau 4. Indicateurs employés lors des analyses Emergy d'après Brown et Ulgiati (1997) modifié par Cavalett et al (2006).

\begin{tabular}{|c|c|c|}
\hline Indicateurs & Expression & Définition \\
\hline Transformité & Y/E & $\begin{array}{l}\text { Emergy totale des intrants sur l'énergie } \\
\text { des produits finaux }\end{array}$ \\
\hline$\%$ d'Emergy renouvelable & $100^{*}\left(R+M_{R}+S_{R}\right) / Y$ & $\begin{array}{c}\% \text { d'Emergy renouvelable sur le total } \\
\text { d'Emergy }\end{array}$ \\
\hline $\begin{array}{c}\text { Taux de charge } \\
\text { environnemental (ELR) } \\
\text { ou } \\
\text { Charge environnementale }\end{array}$ & $\begin{array}{c}(N+F) / R \\
\text { ou } \\
\left(M_{N}+S_{N}+N\right) /\left(R+M_{R}+S_{R}\right)\end{array}$ & $\begin{array}{l}\text { Ratio Emergy des ressources locales non } \\
\text { renouvelables et importees sur Emergy } \\
\text { des sources renouvelables locales }\end{array}$ \\
\hline Rendement Emergy (EYR) & $Y / F$ & $\begin{array}{l}\text { Rendement Emergy par unité d'Emergy } \\
\text { provenant des ressources économiques }\end{array}$ \\
\hline $\begin{array}{c}\text { Rendement d'investissement } \\
\text { Emergy (EIR) }\end{array}$ & $\mathrm{F} / \mathrm{I}$ & $\begin{array}{l}\text { Rendement Emergy provenant des } \\
\text { ressources économiques sur le rendement } \\
\text { des ressources issues de la nature (gratuites) }\end{array}$ \\
\hline Index Emergy de durabilité (EIS) & EYR/ELR & $\begin{array}{l}\text { Ratio soulignant le compromis entre } \\
\text { les avantages du processus étudié } \\
\text { et sa pression sur l'environnement }\end{array}$ \\
\hline
\end{tabular}

ces non renouvelables (locales ou importées/achetées) et l'Emergy issue des sources renouvelables. Cet indicateur sous-entend qu'une fois qu'un service environnemental a été utilisé pour un processus, il n'est plus disponible pour autre chose. Il représente donc le stress qu'exerce le système sur son environnement et permet donc d'appréhender le poids des systèmes agricoles sur l'environnement local (Brown et Ulgiati 1997). Plus la fraction d'Emergy renouvelable est faible, plus la pression environnementale est forte. Cet indicateur est par exemple élevé pour les systèmes à fort niveaux d'intrants. Une valeur inférieure à 2 indique un impact faible sur l'environnement, des valeurs entre 2 et 10 indiquent un impact du système modéré sur l'environnement, des valeurs supérieures à 10 indiquent que le système est à l'origine d'impacts importants dus à des flux importants d'Emergy non renouvelable concentrés dans un environnement relativement restreint. C'est donc un bon indicateur de la durabilité $\mathrm{du}$ processus. Il permet de compléter l'analyse du \% d'Emergy renouvelable.

Le rendement Emergy «Emergy Yield Ratio» (EYR) est le ratio entre l'Emergy totale des intrants (Y) et l'Emergy des intrants provenant de l'activité humaine (F). Il représente la capacité d'un système à exploiter et à rendre disponibles les ressources locales en investissant à plus large échelle, au-delà de ses frontières. L'EYR est donc très sensible à l'utilisation de ressources naturelles $v s$. celle de ressources achetées. Mathématiquement, la valeur minimum de cet indicateur est de 1 ; dans ce cas, le processus de transformation restitue la même quantité d'Emergy que celle qui y est entrée. Il n'y a pas de création d'Emergy, $Y$ étant égal à $\mathrm{F}$ (c'est-à-dire que $\mathrm{I}=0$ ). Le processus ne fait que transformer des ressources déjà existantes et ne participe pas à la création de richesse (Agostinho et al 2008). A titre d'exemple, les sources d'énergie primaire qui sont fortement transformées (ex. gaz, pétrole...) ont un indice généralement supérieur à 5. Les sources d'énergie secondaire et les matériaux primaires (ex. ciment, acier...) ont des indices compris entre 2 et 5 , ce qui indique une contribution modérée à l'économie locale. Les systèmes agricoles intensifs ont une valeur d'EYR inférieure à 2 indiquant ainsi que les fermes ont une forte dépendance vis-à-vis des ressources économiques (Agostinho et al 2008)

Le rendement d'investissement Emergy «Emergy Investment Ratio» (EIR) est une écriture différente de l'EYR $(E Y R=1+1 / E I R)$. Son intérêt réside dans son interprétation parfois plus facile que l'EYR. Il s'agit du ratio entre les intrants achetés (F) et les ressources issues de la nature (renouvelables comme non renouvelables) (I). Il évalue le fait qu'un processus utilise bien ou non l'énergie renouvelable disponible. Un EIR faible caractérise des systèmes efficaces, qui utilisent bien les ressources naturelles disponibles sur le site et peu les ressources payantes.

Enfin, il est possible de combiner EYR et ELR dans un ratio appelé «Emergy Index of Sustainability» (EIS) qui est une mesure agrégée du rendement et de la charge environnementale en tant que fonction de durabilité d'un processus donné. Plus la valeur est élevée, plus le système apparaît comme durable (d'un point de vue Emergy). Avoir un EIS élevé sous-entend soi avoir un EYR élevé soit un ELR faible soit les deux à la fois. Ce qui suppose que le système étudié utilise beaucoup de ressources naturelles (I largement supérieure à F) et peu de ressources non renouvelables ( $\mathrm{R}$ largement supérieur à
$\mathrm{N}$ et $\mathrm{F}$ ). Autrement dit, le système exploite peu de ressources issues de la technosphère, il utilise des ressources naturelles renouvelables, et si son ELR est faible, il provoque peu de stress sur l'environnement.

\section{5 / Analyse de la production de poissons en étang}

Nous avons appliqué la méthode Emergy à un système de production de poissons en étang en région Lorraine (Projet ANR- PISCEnLIT, figure 2 et tableau 3) et nous avons comparé les indicateurs obtenus à ceux de Zhang et al (2011) pour des étangs extensifs et intensifs en Chine (tableau 5). Bien qu'étant tous les trois des systèmes de polyculture (i.e. plusieurs espèces de poissons) en étang, les systèmes diffèrent par leur surface en eau (rapport de 1 à 200 entre les étangs de Zhang et al (2011) et l'étang français), leur rendement, l'alimentation et les espèces cultivées.

Le système français a une transformité plus élevée que les systèmes chinois. Cela indique que le système français est celui des trois systèmes qui a la plus faible efficience. Cette différence entre les systèmes peut s'expliquer par une moins bonne utilisation de l'alimentation (grain de blé) dans le système français en comparaison des deux autres systèmes (consommation de biomasse et de granulés). Il est important de noter que la transformité est sensible à l'efficience du système et non à l'origine des ressources (locales vs. importées, renouvelables $v s$. non-renouvelables). Plus l'EYR est élevé, plus la contribution relative des ressources naturelles (renouvelables ou non) sera élevée. L'étang intensif chinois a une valeur d'EYR proche de 1 (valeur minimale possible pour cet indicateur), alors que l'étang extensif chinois a une valeur de 
Tableau 5. Comparaison d'un étang français avec différents systèmes de production de poissons en étang en Chine (données chinoises issues de Zhang et al 2011).

\begin{tabular}{|c|c|c|c|}
\hline & Etang français & Etang extensif chinois & Etang intensif chinois \\
\hline \multicolumn{4}{|l|}{ Description des systèmes } \\
\hline Zone géographique & $\begin{array}{l}\text { Lorraine, } \\
\text { France }\end{array}$ & $\begin{array}{l}\text { Province du Shangdong, } \\
\text { Chine }\end{array}$ & $\begin{array}{c}\text { Province du } \\
\text { Shangdong, Chine }\end{array}$ \\
\hline Surfaces en eau & 96 ha & 0,67 ha & 0,4 ha \\
\hline Espèces & $\begin{array}{l}\text { Carpes } \\
\text { Sandres } \\
\text { Brochets } \\
\text { Gardons } \\
\text { Tanches } \\
\text { Perches }\end{array}$ & $\begin{array}{l}\text { Carpes Amour } \\
\text { Carpes argentées }\end{array}$ & $\begin{array}{l}\text { Carpes Amour } \\
\text { Carpes argentées } \\
\text { Carpes marbrées }\end{array}$ \\
\hline Quantités produites & $35000 \mathrm{~kg}$ & $4600 \mathrm{~kg}$ & $6650 \mathrm{~kg}$ \\
\hline Rendement (t/ha) & 0,36 & 6,87 & 16,63 \\
\hline Alimentation & Distribution de blé & Distribution d'herbe & $\begin{array}{l}\text { Distribution d'aliment } \\
\text { commercial }\end{array}$ \\
\hline Durée du cycle de production & 11 mois & 8 mois & 8 mois \\
\hline \multicolumn{4}{|l|}{ Indicateurs Emergy } \\
\hline Transformité, sej/J & $6,9 \times 10^{6}$ & $4,6 \times 10^{6}$ & $7,7 \times 10^{5}$ \\
\hline$\% \mathrm{R}$ & 29,0 & 65,0 & 27,0 \\
\hline EYR & 1,52 & 2,19 & 1,04 \\
\hline ELR & 2,45 & 0,55 & 2,73 \\
\hline EIS & 0,62 & 3,98 & 0,38 \\
\hline
\end{tabular}

2,19. Avec une valeur de 1,52, l'étang français apparaît plus proche de l'étang intensif et semble montrer une dépendance plus importante aux ressources économiques. De par sa construction, l'ELR est un indicateur directement relié à la fraction renouvelable des ressources et peut être ainsi considéré comme une mesure du stress sur l'environnement (indication de l'utilisation de ressources non renouvelables ou importées par rapport à l'utilisation de ressources locales renouvelables) due à la production de poissons dans les étangs. Avec un ELR inférieur à $2(0,55)$ l'étang extensif semble avoir peu d'effet sur l'environnement, l'étang français a un impact faible $(\mathrm{ELR}=2,45)$ et l'étang intensif chinois a un impact plus fort $(E L R=2,73)$ bien que restant d'un niveau modéré dans l'absolu. Cependant, cet indicateur ne permet pas de savoir si le type de stress engendré sur l'environnement est local. Enfin, l'EIS des systèmes utilisant de l'aliment (grain de blé pour le système français, aliment composé pour le système intensif chinois) est faible $(0,62$ et 0,38 respectivement) ce qui montre que ces systèmes sont faiblement durables, d'un point de vue Emergy. En effet, ils fournissent un retour d'Emergy faible, au détriment d'une charge environnementale (ELR) relativement élevée.

L'analyse Emergy réalisée sur le système de production en étang français montre, en comparaison avec d'autres systèmes de production de poissons en étang, que le système manque d'efficacité productive par rapport à l'Emergy totale investie, qu'il est dépendant des intrants issus de la technosphère et qu'il ne valorise pas assez d'intrants d'origine renouvelable. A la lumière de ces résultats, il semble intéressant de repenser l'alimentation afin que l'aliment soit mieux valorisé au sein du système.

\section{6 / La méthode Emergy en agriculture}

La méthode Emergy est de plus en plus utilisée en agriculture pour analyser ou comparer les systèmes que ce soit des systèmes de productions végétales, animales ou des systèmes intégrés (polyculture - élevage ou culture poly-élevage). Elle permet aussi d'évaluer l'agriculture à l'échelle d'un pays (Rydberg et Haden 2006).

En productions végétales, la méthode Emergy a été appliquée notamment pour comparer l'effet de différents types de cultures sur l'environnement et l'utilisation de ressources (Lefroy et Rydberg 2003) ou pour comparer différents systèmes de productions végétales (Panzieri et al 2000, Martin et al 2006, Agosthino et al 2008, de Barros et al 2008) afin d'orienter ces systèmes vers des solutions plus durables. Certains auteurs étendent les limites de leur système à la transformation des produits végétaux (production du vin, Bastianoni et al 2001, Pizzigalo et al 2008) ou à la commercialisation des produits (de Barros et al 2008) voire les deux (Cuadra et Rydberg 2006).

L'application de la méthode Emergy en productions animales est moins répandue. La grande majorité des études portent sur les systèmes piscicoles (Odum 2001, Bastianoni 2002, Vassalo et al 2007, Vassalo et al 2009, Zhang et al 2011). Cependant, quelques études existent pour les élevages de volailles et de mammifères. Cavalett et al (2006) ont utilisé la méthode Emergy pour analyser un système intégrant productions végétales, porcs et poissons au Brésil. Dans ce système (Peixe verde), les effluents porcins servent à fertiliser les cultures et à nourrir les poissons. L'analyse Emergy menée à l'échelle du système et à l'échelle de chaque soussystème (culture, porcs, poissons) a permis de mettre en évidence une plus grande efficience des systèmes intégrés comparativement aux systèmes animaux analysés séparément, mais une moindre efficience par rapport à l'atelier végétal. La méthode a pu mettre en évidence l'importance des recyclages de flux au sein des systèmes de productions ayant un ou plusieurs ateliers animaux. Castelini et al (2006) ont comparé un système de production de poulets de chair conventionnel à un système de production biologique en Italie. Très logiquement, le flux total d'Emergy dans le système conventionnel est très largement supérieur ( 8 fois) à celui du système biologique. Cependant, les transformités et certains indicateurs Emergy sont peu différents entre les systèmes, ce qui indique, d'après Castelini et al (2006) les limites du cahier des charges dans les systèmes biologiques en Italie (race peu adaptée, transfert sans adaptation des techniques de l'agriculture conventionnelle). Toutefois, l'étude ne précise pas les hypothèses émises pour le calcul des 
transformités, renforçant ainsi les incertitudes concernant les résultats obtenus. Cette étude souligne la nécessité d'approfondir la recherche sur les systèmes biologiques afin d'optimiser leur durabilité. Une évaluation du système allaitant en Argentine a été menée par Rótolo et al (2007) dans laquelle étaient comparés différents sous-systèmes (prairies naturelles, prairies de fauches, production de céréales d'hiver, production de maïs, production de veaux, engraissement). Cette évaluation montre la faible dépendance aux intrants du sous-système pâturage naturel en comparaison à un sous-système prairies de fauche. Cette étude met aussi en évidence la complexité des relations entre les sous-systèmes d'un système d'élevage que ce soit en termes de synergie ou d'antagonisme entre ces sous-systèmes. Cela souligne la nécessité d'analyser le système dans son ensemble et non comme une addition de différents ateliers.

Ces études mettent en évidence la complexité de mise en œuvre de la méthode Emergy sur les systèmes d'élevage ce qui peut expliquer le peu d'études à ce jour portant sur les systèmes animaux. En effet, ceux-ci nécessitent un certain nombre d'intrants notamment alimentaires (ex. aliments composés, fourrages, pâtures, vitamines...) pour lesquels il est nécessaire de calculer les transformités idoines. Ce qui implique une connaissance de l'origine des matières premières et des itinéraires techniques utilisés pour les obtenir. Cet obstacle apparent à l'utilisation de la méthode Emergy pour les productions animales ne pourra être contourné que par la constitution d'une communauté scientifique travaillant sur ces questions.

\section{Conclusions}

La méthodologie Emergy a été mise au point pour étudier les écosystèmes. Elle paraît donc adaptée pour étudier les agro-écosystèmes que sont les systèmes agricoles. Cette méthode, bien qu'existant depuis 20 ans, connaît un essor particulier depuis ces 5 dernières années dans les domaines agricole et industriel, ce qui renforce son intérêt. Le calcul des transformités reste une étape délicate car ces dernières constituent la clé de voute de cette méthode. L'établissement de bases de données communes pour les transformités est en cours de réalisation. Par rapport à d'autres méthodes d'analyse environnementale, la méthode Emergy permet de resituer un agro-écosystème dans son environnement et d'étudier les interactions avec ce dernier. Cependant, elle ne permet pas de déterminer quel type d'impact potentiel peut se produire sur l'environnement (ex. changement climatique, eutrophisation...) ni de le quantifier comme pourrait le faire d'autres méthodes d'analyse environnementale telle que l'ACV (van der Werf et Petit 2002). Utilisée comme seule méthode d'évaluation, l'Emergy semble insuffisante pour aider à relever les enjeux d'une agriculture écologiquement intensive. En revanche, une combinaison avec l'ACV s'avère intéressante puisque les deux méthodes reposent sur la même logique de construction (définition du système, analyse des flux entrants et sortants) mais ont des finalités différentes. Cette combinaison permettrait de suivre et d'évaluer les changements de pratiques dans les agro-écosystèmes et de les accompagner sur le chemin de l'intensification écologique.

\section{Références}

Agostinho F., Diniz G., Siche R., Ortega E., 2008. The use of emergy assessment and the geographical information system in the diagnosis of small family farms in Brazil. Ecol. Model., 210, 37-57.

Bastianoni S., 2002. Use of thermodynamic orientors to acess the efficiency of ecosystems: A case study in the lagoon of venise. Sci. World J., 2, 255-260.

Bastianoni S., Marchettini N., 2000. The problem of co-production in environmental accounting by emergy analysis. Ecol. Model., 129, 187-193.

Bastianoni S., Marchettini N., Panzieri M., Tiezzi E., 2001. Sustainability assessment of a farm in the chianti area (Italy). J. Cleaner Prod., 9, 365-373.

Bergquist D.A., 2008. Colonised coasts. Aquaculture and emergy flows in the world system: Cases from Sri Lanka and the Philippines, Uppsala Universitet, Sweeden, 186pp.

Brandt-Williams S.L., 2002. Handbook of emergy evaluation: A compendium of data for emergy computation issued in a series of folios. Folio \# 4 - emergy of florida agriculture. Center for environmental policy, environmental engineering sciences, University of Florida, Gainesville, Floride, 40pp. Available in: http://emergysystems.org/ folios.php

Brown, M.T., Ulgiati, S., 1997. Emergybased indices and ratios to evaluate sustainability: Monitoring economies and technology toward environmentally sound innovation. Ecol. Eng., 9, 51-69.
Brown M.T., Ulgiati S., 2004. Energy quality, emergy, and transformity: H.T. Odum's contributions to quantifying and understanding systems. Ecol. Model., 178, 201-213.

Castellini C., Bastianoni S., Granai C., Da Bosco A., Brunetti M., 2006. Sustainability of poultry production using the emergy approach: Comparison of conventional and organic rearing systems. Agric. Ecosyst. Env., 114, 343350 .

Cavalett O., de Queiroz J.F., Ortega E., 2006. Emergy assessment of integrated production systems of grains, pig and fish in smal farms in the South Brazil. Ecol. Model., 193, 205-224.

Cavalett O., De Queiroz J.F., Ortega E. 2007. Emergy accounting of fish aquaculture chains in Brazil. Biol. Ital. Ecodinamica, 7, 5361.

Cuadra M., Rydberg T., 2006. Emergy evaluation on the production, processing and export of coffee in nicaragua. Ecol. Model., $196,421-433$

de Barros I., Blazy J.M., Rodrigues G.S., Tournebize R., Cinna J.P., 2008. Emergy evaluation and economic performance of banana cropping systems in Guadeloupe (French west indies). Agric. Ecosyst. Env., 129, 437-449.

Griffon M., 2010. Pour des agricultures écologiquement intensives des territoires à haute valeur environnementale et de nouvelles politiques agricoles, Éd. de l'Aube (Monde en cours), La Tour d'Aigues, France.
Haukoos D.S., 1995. Sustainable architecture and its relationship to industrial building. Masters thesis, University of Florida, Gainsville, Floride, 276p.

Landais E., 1987. Recherches sur les systèmes d'élevage. Questions et perspectives. Document de travail de l'URSAD V.D.D, INRA-SAD, Versailles, France, 75p.

Lefroy E., Rydberg T., 2003. Emergy evaluation of three cropping systems in southwestern australia. Ecol. Model., 161, 195-211.

Martin J.F., Diemont S.A.W., Powell E., Stanton M., Levy-Tacher S., 2006. Emergy evaluation of the performance and sustainability of three agricultural systems with different scales and management. Agric. Ecosyst. Env., 115, 128-140.

Odum H.T., 1983. Systems Ecology: An introduction. Wiley, New York, 644p.

Odum H.T., 1996. Environmental accounting: Emergy and environmental decision making. Wiley, New York, USA, 370p.

Odum H.T., 2000. Handbook of emergy evaluation: A compedium of data for emergy computation issued in a series of folios. Folio \# 2 - emergy of global processes. Center for environmental policy, environmental engineering sciences, University of Florida, Gainesville, Floride, 28pp. Available in:http://emergysystems.org/folios.php

Odum H.T., 2001. Emergy evaluation of salmon pen culture. Environmental engineering 
sciences. University of Florida, Gainesville, Floride, 9p.

Ortega E., 2008. 2nd Seminário de Aqüicultura Sustentável. CA-UNESP, Jaboticabal, São Paulo, Bresil, 28-29.

Panzieri M., Marchettini N., Hallam T.G., 2000. Importance of the bradhyrizobium japonicum symbiosis for the sustainability of asoybean cultivation. Ecol. Model., 135, 301-310.

Paoli C., Vassallo P., Fabiano M., 2008. Solar power: an approach to transformity evaluation. Ecol. Eng., 34, 191-206.

Pizzigallo A.C.I., Granai C., Borsa S., 2008. The joint use of lca and emergy evaluation for the analysis of two italian wine farms. J. Env. Manag., 86, 396-406.
Rotolo G.C., Rydberg T., Lieblein G., Francis C., 2007. Emergy evaluation of grazing cattle in argentina's pampas. Agric. Ecosyst. Env., $119,383-395$.

Rydberg T., Haden A.C., 2006. Emergy evaluations of Denmark and Danish agriculture: Assessing the influence of changing resource availability on the organization of agriculture and society. Agric. Ecosyst. Env., 117, 145-158.

Ulgiati S., Brown M.T., 2002. Quantifying the environmental support for dilution and abatement of process emissions - the case of electricity production. J. Cleaner Prod., 10, 335-348.

van der Werf H.M.G., Petit J., 2002. Evaluation of the environmental impact of agriculture at the farm level: a comparison and analysis of 12 indicator-based methods. Agric. Ecosyst. Env., 93, 131-145.

Vassallo P., Bastianoni S., Beiso I., Ridolfi R., Fabiano M., 2007. Emergy analysis for the environmental sustainability of an inshore fish farming system. Ecol. Indicators, 7, 290-298.

Vassallo P., Beiso I., Bastianoni S., Fabiano M., 2009. Dynamic emergy evaluation of a fish farm rearing process. J. Environ. Manag., 90, 2699-2708.

Zhang L.X., Ulgiati S., Yang Z.F., Chen B., 2011. Emergy evaluation and economic analysis of three wetland fish farming systems in Nansi Lake area, China. J. Env. Manag., 92, 683-694.

\title{
Résumé
}

L'Emergy est définie comme la somme de toutes les formes d'énergie (énergie directe et indirecte, renouvelable et non-renouvelable) nécessaire à la réalisation d'un service ou d'un produit. La méthode d'évaluation Emergy est une méthode d'analyse quantitative qui détermine la valeur non-monétaire et monétaire des ressources, des services et des produits dans une unité commune : l'Emergy solaire. Cette méthode apparaît comme intéressante pour évaluer les agro-écosystèmes et identifier des leviers d'action pour la conception de systèmes plus durables. Cet article présente les concepts de la méthode Emergy en explicitant les notions clés telles que la transformité, le diagramme de hiérarchisation de l'énergie et les indicateurs Emergy. Ces notions sont explicitées en se basant sur l'exemple d'une analyse de la production de poissons en étang. Enfin, l'article détaille les applications possibles de la méthode pour les systèmes agricoles et notamment en production animale.

\begin{abstract}
Emergy accounting: principles and application for environmental assessment of agricultural and animal production systems

Emergy is defined as the direct and indirect energy available to make a product or a service. Emergy accounting is a quantitative technique that determines non-monetary and monetary values of resources, services and products in a common unit: solar Emergy. This method appears suitable to assess agroecosystems and identify key factors for designing more sustainable systems. This article discusses the concept of Emergy through the definition of transformity, energy-system diagrams and several Emergy indicators. These concepts are explained through an example of a fish-farming system assessment. Finally, the article underlines the possible applications of emergy accounting to agricultural systems, in particular animal production.
\end{abstract}

WILFART A., CORSON M.-S., AUBIN J., 2012. La méthode EMERGY : principes et application en analyse environnementale des systèmes agricoles et de production animale. INRA Prod. Anim. 25, 57-66. 